\title{
Duodenum sparing technique for preventive stenting of biliary tract anastomosis in pigs
}

\author{
Tomáš Grolich ${ }^{1}$, Michal Crha $^{2}$, Tomáš Andrašina ${ }^{3}, Z_{\text {Zdeněk Kala }}{ }^{1}$, Petr Raušer ${ }^{2}$, \\ Jan Hlavsa ${ }^{1}$, Martin Potrusil ${ }^{1}$ \\ ${ }^{1}$ Masaryk University, University Hospital Brno and Faculty of Medicine, \\ Department of Surgery, Brno, Czech Republic \\ ${ }^{2}$ University of Veterinary and Pharmaceutical Sciences Brno, Faculty of Veterinary Medicine, \\ Small Animal Clinic, Brno, Czech Republic \\ ${ }^{3}$ Masaryk University, University Hospital Brno and Faculty of Medicine, \\ Department of Radiology and Nuclear Medicine, Brno, Czech Republic \\ Received February 7, 2019 \\ Accepted June 13, 2019
}

\begin{abstract}
The main aim of this study was to find a standardized method for preventive stenting of biliary tract anastomosis with biodegradable self-expandable stent from polydioxanone and to evaluate the functionality of the stent. The experimental study was conducted using four pigs with a follow-up of eight weeks. The procedure was done under general anaesthesia. The function of the stent was verified by clinical stage, blood test, magnetic resonance cholangiopancreatography and necropsy. Our duodenum sparing technique was described in detail and photographically documented. All pigs finished the follow-up period without clinical, laboratory or radiologic signs of biliary obstruction. Necropsy did not reveal complication of the procedure or anastomotic stricture. In conclusion, this study demonstrated a simple new duodenum sparing method of transanastomotic insertion of a biodegradable self-expandable stent from polydioxanone. We did not find any complications during the follow-up. This method will be used in our follow-up study with an extended experimental control group of animals and a longer follow-up period to verify the preventive functionality of this stent.
\end{abstract}

\section{Biodegradable, self-expandable stent, anastomotic stricture, polydioxanone}

A benign stenosis or stricture of the biliary tract anastomosis is a clinical problem which has its consequences in recurrent cholangitis and the possible development of secondary biliary cirrhosis, often in immunosuppressed transplanted patients.

In contemporary medicine, we have some opportunities to diagnose and treat this complication by dilatation and stenting via endoscope, transhepatic drainage or by surgical procedure as the last step. Only few authors have focused on the prevention of a stricture during surgery in their studies (Luo et al. 2013; Shi et al. 2013; Janousek et al. 2016). As a solution to the disadvantages connected with the placement, occlusion, migration and extraction of the commonly used plastic or more recently covered metallic stent, the biodegradable self-expandable polydioxanone stent has been studied in a preventive setting.

Based on our previous experimental study dealing with the biodegradable self-expandable stent in the biliary tree which observed its biocompatibility during biodegradation (Grolich et al. 2015), we decided to follow up with its use in the transanastomotic setting. The main aim of this study was to find a safe and simple method of transanastomotic stent delivery during its surgical construction without the need of duodenotomy. The evaluation of the stent function by blood tests, magnetic-resonance cholangiopancreatography (MRCP) and necropsy was planned as the secondary aim.

Address for correspondence: 


\section{Materials and Methods}

\section{Stent and the delivery system}

The braided stents were manufactured from commercially available polydioxanon monofilament EP 3 fibre (European Pharmacopoeia; $\mathrm{n}$ EP $=\mathrm{n} * 0.1 \mathrm{~mm}$; EP $3=0.3 \mathrm{~mm}=$ USP 2-0). All stents were handmade according to our requirements (Ella DV biliary stent, Ella-CS, s.r.o., Hradec Králové, Czech Republic). The sterile stent was packed separately from the delivery system because of its plastic deformation and was loaded manually into the delivery system just before the implantation (Plate I, Figs 1,2).

\section{Animal care and the anaesthetic protocol}

The whole study was carried out upon the approval by the Ethics Committee of the University of Veterinary and Pharmaceutical Sciences in Brno and by the Ministry of Health (reg. no. 43-2015) according to the applicable law on experimental animal protection. Four white domestic pigs (aged 3 months; weight, $30-35 \mathrm{~kg}$, gilts only, because of their different anatomy during the midline laparotomy) were used for implantation of the intra-biliary stent. The animals were housed in a stable of the Veterinary Research Institute, Brno, Czech Republic. The implantation was performed at the Department of Surgery of the Faculty of Veterinary Medicine, University of Veterinary and Pharmaceutical Sciences in Brno, Czech Republic. Pigs were fed at a basic rate with an all-day access to water. The pigs were anaesthetized using the following protocol: tiletamine $2 \mathrm{mg} / \mathrm{kg}+$ zolazepam $2 \mathrm{mg} / \mathrm{kg}$ (Zoletil 100, Virbac, France) + ketamine $2 \mathrm{mg} / \mathrm{kg}$ (Narketan, Vetoquinol, France) + xylazine $2 \mathrm{mg} /$ $\mathrm{kg}$ (Sedazine, Fort Dodge, USA) i.m. for introduction, followed by the placement of an i.v. canula and the administration of propofol (Propofol, Fresenius, Germany) $2 \mathrm{mg} / \mathrm{kg}$ i.v., placement of a tracheal canula and conduction of anaesthesia by isoflurane (Aerrane, Baxter, USA) and oxygen.

\section{Surgical technique}

Midline upper laparotomy was performed after standard aseptic preparation of the surgical field. After the exposure of the common bile duct and preparation of a $2 \mathrm{~cm}$ long segment of it (Plate I, Fig. 3), a transection followed in the middle of the liberalized segment with measurement of the proximal lumen diameter with a biliary Bakeš probe (Medin, Nové Město na Moravě, Czech Republic) (Plate I, Fig. 4) and followed by the construction of the dorsal part of end-to-end anastomosis (Plate I, Fig. 5) with continuous suture using a biodegradable stitch - 5/0 polydioxanone (Monoplus, BBraun, Melsungen, Germany). Then a guidewire from the central venous catheter (Certofix Trio, BBraun, Melsingen, Germany) of a $0.89 \mathrm{~mm}$ diameter was introduced with its straight tip through the anastomotic site and the duodenal papilla into the duodenum against its wall (Plate I, Fig. 6) and a small puncture wound was performed by electrocautery to access the tip of the guidewire. The wire was placed with its flexible J-tip as high as possible into the biliary tree and the ventral part of anastomosis was finished with the same stitch. After the completion of anastomosis, the stent in its delivery system was placed through the anastomosis using a dilatator (Plate I, Figs 7, 8) and released. The position of anastomosis approximately in the middle of the stent and out of the duodenal lumen was kept in check (Plate II, Fig. 9). The puncture wound of the duodenum was closed by one stitch with 5/0 Monoplus (Plate II, Fig. 10). Laparotomy was closed using the monofilament suture; the peritoneum with 1/0 polyglactine (Novosyn, BBraun, Melsungen, Germany), the fascia and subcutaneous tissue separately with 1/0 polydioxanone (Monoplus, BBraun, Melsungen, Germany), and the skin with 3/0 polyamide (Dafilon, BBraun, Melsungen, Germany). Animals were checked daily for their clinical status. Postoperative analgesia was administered by i.m. application of buprenorfin (Temgesic, Schering-Plough, Kenilworth, USA) at a dose of $0.01 \mathrm{mg} / \mathrm{kg}$.

The experimental animals were followed up for 8 weeks and investigated by MRCP at the end of the follow-up period under general anaesthesia, and after that euthanized with an i.v. injection of T61 (200 mg embutramide, $50 \mathrm{mg}$ mebozonium iodide and $5 \mathrm{mg}$ tetracaine hydrochloride) which was preceded by i.m. sedation with medetomidin (Domitor, Orion CorporationOrion Corporation, Finland) and followed by necropsy. Blood tests including the complete blood count (Sysmex XT-2000iV, Sysmex Corporation, Kobe, Japan), serum bilirubin and liver tests for alanine transaminase, aspartate transaminase, alkaline phosphatase, gamma-glutamyltransferase (Abbott Architect c4000, Abbott Laboratories, IL, USA), at the time of the operation and before euthanasia were carried out. All data were analyzed using basic descriptive statistics.

\section{Results}

All stents were inserted through the anastomosis easily. Self-expansion was correct in all cases. Clinical symptoms of bile duct obstruction were absent and no animal suffered from any surgical procedure complication in the biliary tree or the duodenum. The clinical status of the pigs in the postoperative period was good except for a lower appetite within two days immediately after the surgery. Euthanasia of the experimental animals was performed according to the established schedule. 
Blood tests did not reveal any pathological findings in the leukocyte levels, serum bilirubin levels and in all liver tests in all pigs before the procedure and at the end of the follow-up period.

Magnetic-resonance cholangiopancreatography showed patency of the stent in all pigs after 8 weeks and the anastomotic stricture or dilatation of the proximal biliary tree was absent (Plate II, Fig. 11).

Necropsy showed normal healing within the abdominal cavity except for a ventral hernia and adhesions to the laparotomy in two pigs. No cholangitis, necrosis, abscess or excessive fibroplasia of bilioma was found in the hepato-duodenal ligament during the final necropsy. Macroscopic evaluation of bile ducts showed a lightening of the stent's blue colour and its partial disintegration (Plate II, Fig. 12). Stenosis measured by the Bakeš probe was not found in the anastomotic site. (Plate II, Fig. 13)

\section{Discussion}

This study details our technique of duodenum sparing of transanastomotic stent placement. Our procedure allowed effortless placement of the stent through the duodenal puncture, papilla, and across the common bile duct anastomosis after its construction with correct self-expansion in all pigs. In their experimental study in rabbits, Chen et al. (2013) used different stents placed via a guide wire with the need of duodenotomy as described also in our previous study (Grolich et al. 2015). One clinical study referred to the preventive use of the same but shorter stent in transplanted patients. A stenting technique across anastomosis was not described (Janoušek et al. 2016). Shi et al. (2013) presented a study on the preventative stenting of biliary anastomosis with a completely different type of short stent covered with chemotherapeutics placed through the anastomosis without the need of tensile strength. Tanimoto et al. (2016) inserted a stent (made by copolymerization of L-lactide and $\varepsilon$-caprolactone and coated with barium sulphate) into the transected common bile duct and secured it with 6-0 polydioxanone absorbable monofilament suture. This was the only experimental study we found dealing with the preventive trans-anastomotic stenting with a radiologic (computed tomography) follow-up.

We have not found any other study on the surgical placement of a self-expandable biodegradable stent into the common bile duct anastomosis during its construction. This technique can be used in similar anastomoses in experimental animals or humans with the same or equally constructed stent and delivery system.

Our results after eight weeks of follow-up suggest that this procedure is safe in terms of biliary obstruction as verified by blood tests and necropsy. Our previous study revealed that the time of complete degradation of this stent was at least 13 weeks without cholangitis in the period of 22 weeks of follow-up (Grolich et al. 2015). Small parts of the stent are eliminated through the duodenal papilla and could be the reason for the transient mild cholangitis observed in clinical studies in patients after the therapeutic stenting of a benign biliary stricture (Petrtýl et al. 2010; Mauri et al. 2013).

None anastomotic stenosis was found during the MRCP and necropsy of the biliary tree. All stents were patent during the MRCP and found during the dissection. We did not find any study using MRCP to detect complications after the preventive stenting of the biliary tree or its anastomosis. This method and all these promising findings will be used in our follow-up study with the extended experimental control group of animals and longer follow-up to verify the preventive function of this stent.

\section{Acknowledgements}

Our study was supported by the Ministry of Health Czech Republic, project AZV, reg. no.: 15-32484A. 


\section{References}

Grolich T, Crha M, Novotný L, Kala Z, Hep A, Nečas A, Hlavsa J, Mitas L, Misik J 2015: Self-expandable biodegradable biliary stents in porcine model: The Initial Experience. J Surg Res 193: 606-612

Chen YG, Yan J, Wang ZG, Yu S, Yuan ZM, Wang XH, Zhang XN, Zheng Q 2013: Technique for the safe placement of a biodegradable stent into the common bile duct of rabbits: The initial experience. Exp Ther Med 6: 1101-1104

Janousek L, Maly S, Oliverius M, Kocik M, Kucera M, Fronek J 2016: Bile duct anastomosis supplied with biodegradable stent in liver transplantation: the initial experience. Transplant P 48: 3312-3316

Luo Z, Cheng L, Ren J, Tang L, Wang T, Tian ZF 2013: Progressive balloon dilatation following hepaticojejunostomy improves outcome of bile duct stricture after iatrogenic biliary injury. BMC Gastroenterol 13: 70

Mauri G, Michelozzi C, Melchiorre F, Poretti D, Tramarin M, Pedicini V, Solbiati L, Cornalba G, Sconfienza LM 2013: Biodegradable biliary stent implantation in the treatment of benign bilioplastic-refractory biliary strictures: preliminary experience. Eur Radiol 23: 3304-3310

Petrtýl J, Bruha L, Horak L, Zadorova Z, Dosedel Z, Laash HU 2010: Management of benign intrahepatic bile duct strictures: initial experience with polydioxanone biodegradable stents. Endoscopy 42 (Suppl. 2): E89-E90

Shi JH, Lv Y, Yu L, Zhang BB, Zhang XF, Fan C, Geng ZM 2013: Interest of a new biodegradable stent coated with paclitaxel on anastomotic wound healing after biliary reconstruction Eur J Gastroenterol Hepatol 25: $1415-1423$

Tanimoto Y, Tashiro H, Mikuriya Y, Kuroda S, Hashimoto M, Kobayashi T, Taniura T, Ohdan H 2016: Radiopaque biodegradable stent for duct-to-duct biliary reconstruction in pigs. Langenbeck Arch Surg 401: 513-517 
Plate I

Grolich T. et al.: Duodenum sparing ... pp. 287-290

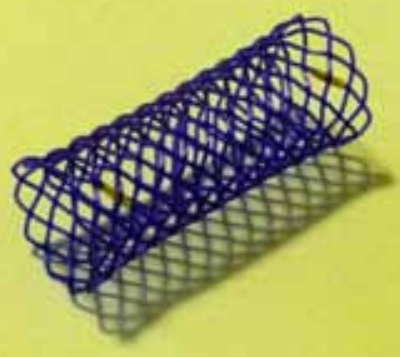

Fig. 1. The polydioxanon self-expandable stent, $10 \times 30 \mathrm{~mm}$

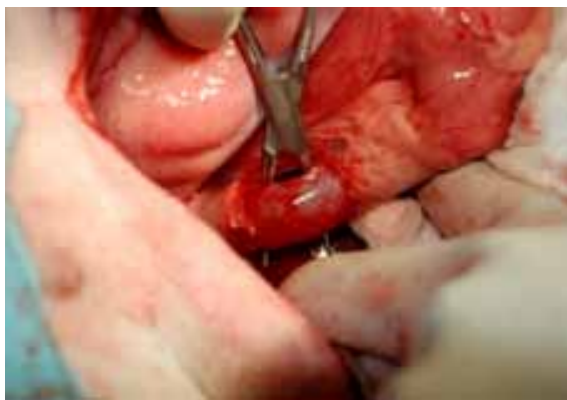

Fig. 3. Common bile duct preparation

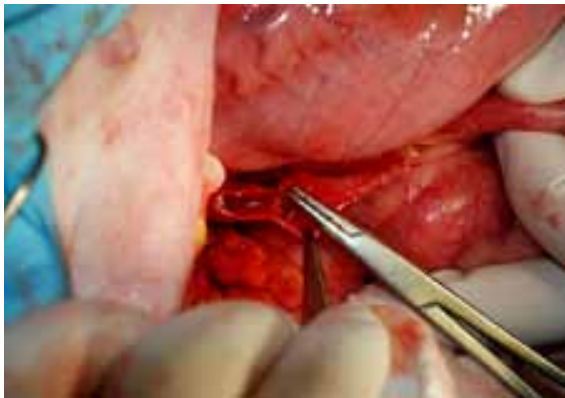

Fig. 5. Construction of the dorsal part of end-to-end anastomosis

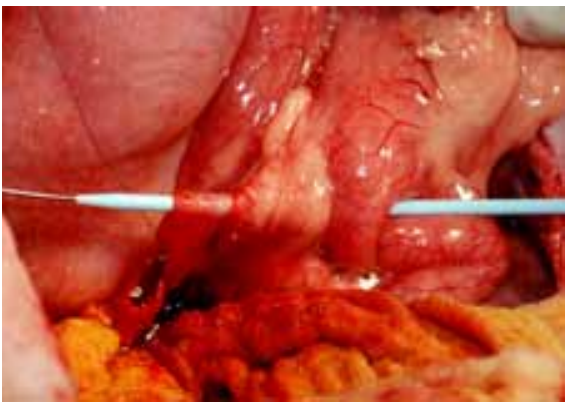

Fig. 7. Dilatation of the duodenal papilla via the guide wire

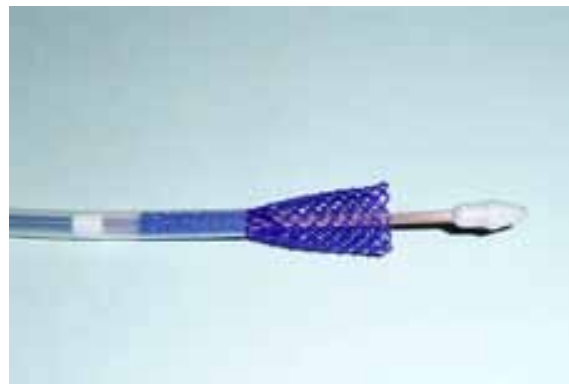

Fig. 2. Stent loading into the delivery system

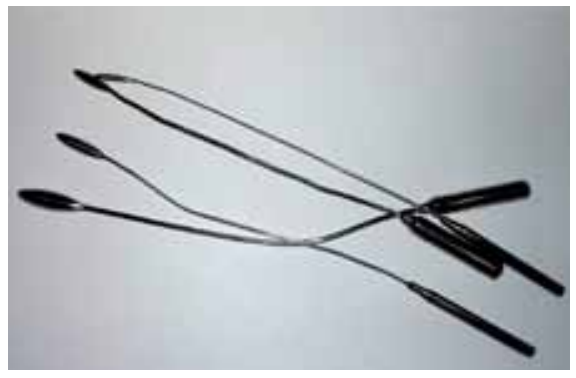

Fig. 4. The Bakeš probes

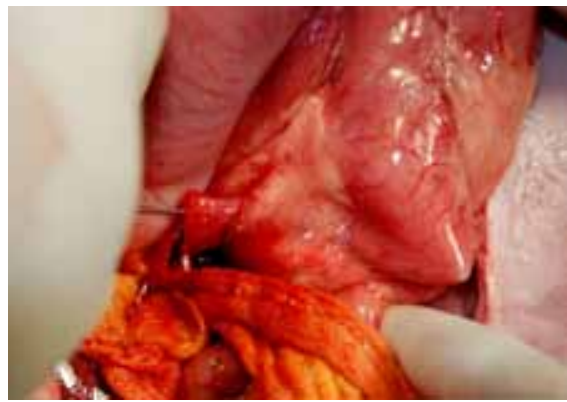

Fig. 6. Retrograde puncture of the duodenal wall by the guide wire

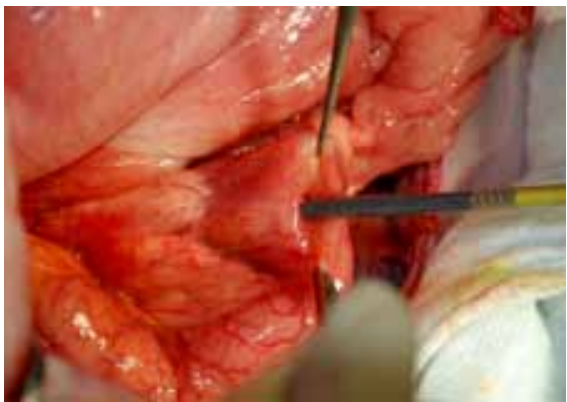

Fig. 8. Placement of the stent delivery system through anastomosis via the guide wire 
Plate II

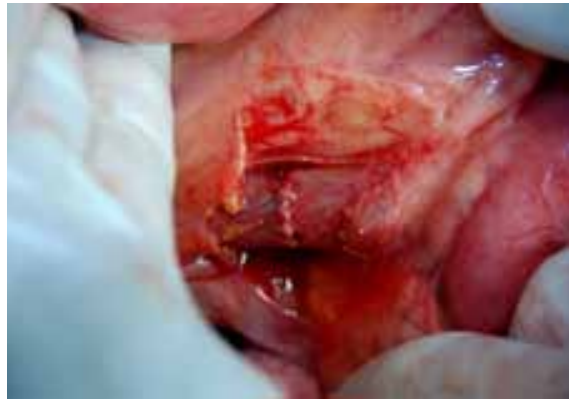

Fig. 9. Checking the position after a full selfexpansion of the stent

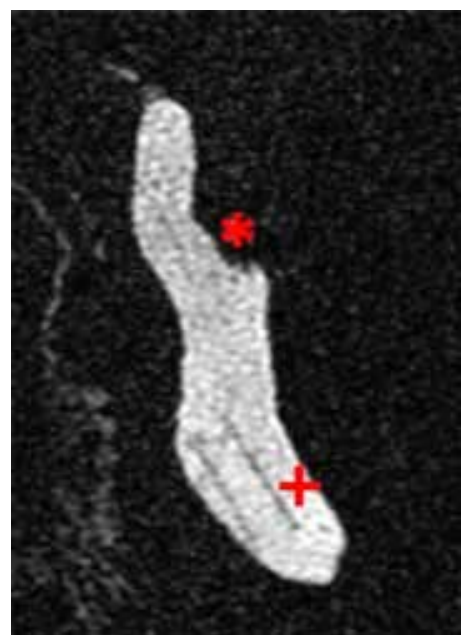

Fig. 11. The MRCP reconstruction after 8 weeks, showing the common bile duct and its division $(*)$ with patency of the stent in the lumen $(+)$. Anastomotic site of stenosis on the common bile duct is not visible.

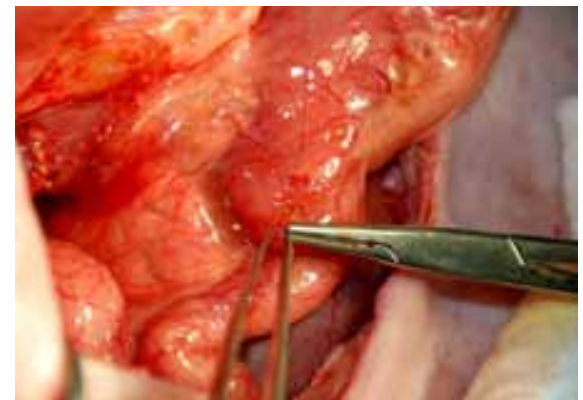

Fig. 10. Closure of the duodenal defect

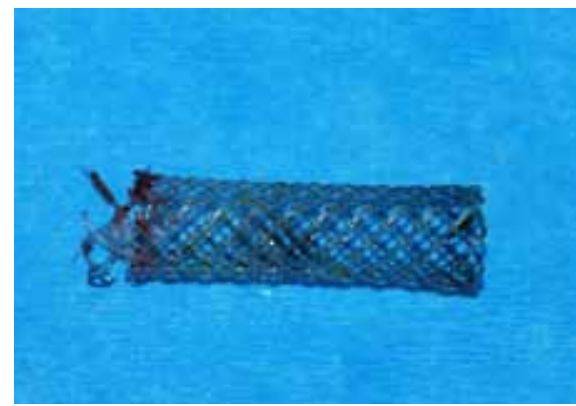

Fig. 12. The stent extracted during necropsy with a visible lightening of its blue color and partial disintegration.

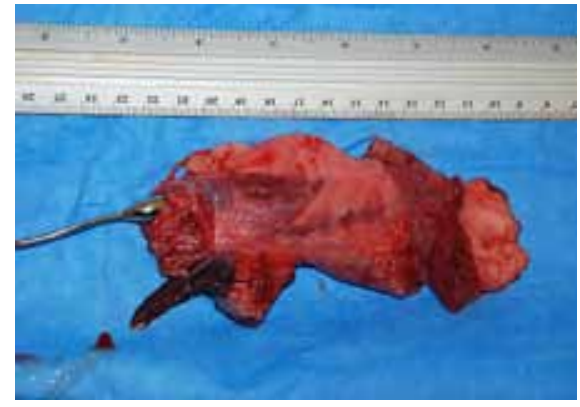

Fig. 13. Extrahepatic biliary tree extracted during necropsy with its diameter measured using the Bakeš probe. 